\title{
A percepção do enfermeiro acerca da desospitalização em cuidados paliativos
}

\begin{abstract}
RESUMO
Este estudo tem como objetivos descrever e analisar a visão do enfermeiro como integrante da equipe multiprofissional de uma unidade hospitalar, acerca do processo de desospitalização da pessoa com câncer em cuidados paliativos. A metodologia deste trabalho consiste em uma pesquisa descritiva e com abordagem qualitativa, sendo o cenário para a coleta de dados o Hospital Federal Cardoso Fontes nas unidades de Internação Clínica, Cirúrgica e de Emergência e os participantes os enfermeiros lotados nestes setores. A análise de dados seguiu as três fases apresentadas para a análise de conteúdo, originando duas categorias temáticas: A visão do enfermeiro, como membro da equipe multiprofissional acerca dos cuidados paliativos e desospitalização e Possibilidades e Limites do processo de desospitalização da pessoa com câncer em cuidados paliativos. Este trabalho levou à percepção da existência de uma lacuna teórica ou acadêmica que os profissionais entrevistados apresentam sobre o tema, demonstrando que ainda falta uma base forte teórica sobre a temática que lhes permita nortear melhor suas práticas no dia a dia. DESCRITORES: Neoplasia; Cuidados Paliativos; Atendimento Domiciliar; Enfermagem Oncológica; Cuidados de Enfermagem.
\end{abstract}

\section{ABSTRACT}

This study aims to describe and analyze the nurse's view as part of the multiprofessional team of a hospital unit, about the process of dehospitalization of the person with cancer in palliative care. The methodology of this work consists of a descriptive research with a qualitative approach, and the scenario for data collection is the Federal Cardoso Fontes Hospital in the Clinical, Surgical and Emergency inpatient units and the participants the nurses in these sectors. Data analysis followed the three phases presented for content analysis, giving rise to two thematic categories: The nurse's view as a member of the multidisciplinary team about palliative care and dehospitalization and Possibilities and Limits of the process of dehospitalization of cancer patients in care palliative. This work led to the perception of the existence of a theoretical or academic gap that the interviewed professionals present on the subject, demonstrating that there is still a lack of strong theoretical base on the theme that allows them to better guide their daily practices.

KEYWORDS: Neoplasia; Palliative Care; Home Care; Oncologic Nursing; Nursing Care.

\section{RESUMEN}

Este estudio tiene como objetivo describir y analizar la opinión de la enfermera como parte del equipo multiprofesional de una unidad hospitalaria, sobre el proceso de deshospitalización de la persona con cáncer en cuidados paliativos. La metodología de este trabajo consiste en una investigación descriptiva con un enfoque cualitativo, y el escenario para la recolección de datos es el Hospital Federal Cardoso Fontes en las unidades de hospitalización clínica, quirúrgica y de emergencia y los participantes, las enfermeras en estos sectores. El análisis de datos siguió las tres fases presentadas para el análisis de contenido, dando lugar a dos categorías temáticas: La opinión de la enfermera como miembro del equipo multidisciplinario sobre cuidados paliativos y deshospitalización y Posibilidades y límites del proceso de deshospitalización de pacientes con cáncer en atención. Paliativos. Este trabajo llevó a la percepción de la existencia de una brecha teórica o académica que los profesionales entrevistados presentan sobre el tema, lo que demuestra que todavía existe una base teórica sólida sobre el tema que les permite guiar mejor sus prácticas diarias.

PALABRAS CLAVE: Neoplasia; Cuidados Paliativos; Cuidado en el Hogar; Enfermería Oncológica; Cuidados de Enfermería.

RECEBIDO EM: 21/11/2019 APROVADO EM: 22/11/2019

\section{Fabiana Andrade Pesso}

Enfermeira. Especialista nos moldes de residência em Clínica Médica e Cirúrgica pela UNIRIO.

\section{Daniel Azevedo da Fonseca}

Enfermeiro. Especialista nos moldes de residência em Clínica Médica e Cirúrgica pela UNIRIO. 


\section{artigo}

Pesso, F.A.; Fonseca, D.A.; Olário, P.S.; Pinto, A.C.S.; Moreira, M.C.; Pestana. L.C.; Neves, K.C.;

A percepção do enfermeiro acerca da desospitalização em cuidados paliativos

\section{Patrícia da Silva Olário}

Enfermeira. Doutora e Mestre em Enfermagem pela Escola de Enfermagem Anna Nery, da Universidade Federal do Rio de Janeiro (EEAN/UFRJ).

\section{Ana Cristina Silva Pinto}

Enfermeira. Doutora e Mestre em Enfermagem. Professora Adjunta II da Universidade Federal do Estado do Rio de Janeiro do Departamento de Enfermagem Médico-cirúrgico da Escola de Enfermagem Alfredo Pinto- UNIRIO.

\section{Marléa Chagas Moreira}

Enfermeira. Doutora e Mestre em Enfermagem. Professora Associada do Departamento de Metodologia da Enfermagem da Escola de Enfermagem Anna Nery/UFRJ.

\section{Luana Cardoso Pestana}

Enfermeira. Mestre em Ciências do Cuidado em Saúde pela Universidade Federal Fluminense.

\section{Keila do Carmo Neves}

Enfermeira. Doutora e Mestre em Enfermagem pela Escola de Enfermagem Anna Nery, da Universidade Federal do Rio de Janeiro (EEAN/UFRJ).

\section{INTRODUÇÃO}

$\mathbf{A}$ desospitalização vem sendo tema de discussões em diversos pontos no Brasil, sobretudo, no que tange à prestação de cuidados à pessoa com câncer em paliação, sendo citada como um dos eixos centrais da atenção domiciliar pelo Ministério da Saúde ${ }^{(1)}$.

Esta transição do cuidar do hospital para o domicílio vem sendo apontada pela literatura ${ }^{(2,3)}$ como um processo denominado desospitalização, tendo papel de interlocutor com diferentes pontos de atenção à saúde, promovendo à pessoa e família protagonismo nas decisões e construção do cuidado de forma coletiva e integral, sendo o enfermeiro o membro da equipe multiprofissional capaz de minimizar as incertezas que possam surgir, pactuar metas em conjunto e otimizar as ações do cuidar em saúde.

Assim, a busca por compreender a visão do enfermeiro, como membro da equipe multiprofissional, frente ao processo de desospitalização da pessoa com câncer em cuidados paliativos, emerge da vivência de dois residentes em enfermagem no Curso de Pós-Graduação em nível de Especialização sob a forma de treinamento em serviço nos moldes de residência, lotados no Hospital Federal Cardoso Fontes, Rio de Janeiro/RJ.

Assim, as experiências vividas pelos residentes neste cotidiano do cuidar frente ao processo de desospitalização da pessoa com câncer em cuidados paliativos, bem como a percepção acerca das situações e conflitos diante das questôes de paliação e transição do cuidar culminaram na inquietação para a elaboração desta pesquisa.

$\mathrm{O}$ enfermeiro inserido no contexto da paliação, sobretudo na Oncologia, precisa entender a pessoa portadora de uma doença ameaçadora da vida, os seus significados e conceitos, bem como considerar a abordagem multiprofissional, os diferentes espaços do cuidar, a autonomia do sujeito e refletir acerca da sua inserção neste universo, buscando resolver questóes que abarquem os aspectos físicos, psíquicos, sociais e espirituais identificados, podendo ou não essa pessoa estar em fase de finitude ${ }^{(4,5)}$.

Neste contexto, deve-se refletir para além do cuidado hospitalocêntrico, pensar a temática desospitalização em cuidados paliativos requer entender a transição do cuidado do hospital para o domićlío como sendo um processo que envolve uma multidimensionalidade, que precisa romper com paradigmas, buscando a possibilidade de cuidar em diversos cenários, englobando múltiplos saberes que se complementam na teia complexa da paliação e da abordagem multiprofissional, tornando a pessoa com câncer e família o centro deste processo e o enfermeiro um facilitador e articulador entre os diversos elementos envolvidos neste universo.
Assim, estar aberto ao "novo" requer ultrapassar a esfera do cuidar pautada em um único local, direcionado aos saberes formais, fragmentados e distanciados da inserção da pessoa e família neste contexto.

Para isso, a reformulação do pensamento profissional, revendo os seus próprios conceitos e olhares acerca deste fenômeno, proporciona a construção de novas ideias e saberes constituídos de modo que possa agregar novas maneiras e atitudes frente ao cuidado prestado, considerando-se a multidimensionalidade e pluralidade pela qual perpassa o contexto da desospitalização e dos cuidados paliativos da pessoa com câncer, bem como o papel do enfermeiro dentro da equipe multiprofissional.

Neste sentido, este estudo se justifica em virtude do aumento de casos de pessoas com doença oncológica no Brasil ${ }^{(5)}$, bem como a precariedade de estudos científicos que abordem a temática desospitalização e cuidados paliativos, sendo necessárias novas reflexões acerca deste contexto, buscando as melhorias da prática de cuidados para a enfermagem e afirmação do seu protagonismo junto à equipe multiprofissional, ampliando e melhorando a sua forma de abordagem, otimizando fluxos e rotinas organizacionais pautadas nas fundamentações científicas que englobam estas temáticas.

Deste modo, acredita-se ser possível o enfermeiro olhar para a sua prática e 
perceber as potencialidades e fragilidades encontradas no cenário estudado, visando açôes que possibilitem contemplar a crescente demanda da população em cuidados paliativos, a partir do aumento do envelhecimento populacional, das doenças crônicas e avanço da patologia oncológica, encarando a desospitalização como sendo uma estratégia para a adoção de novas formas de cuidar desta população( ${ }^{(6)}$.

Cabe, ainda no contexto do cuidar frente às doenças incuráveis, considerar aspectos que garantam o conforto em seus diversos aspectos, bem como as vontades da pessoa e família nos diferentes espaços e contextos que englobam a paliação ${ }^{(7)}$.

Assim, os objetivos deste estudo foram identificar as situaçóes-problema reconhecidas pelo enfermeiro no processo de desospitalização em cuidados paliativos oncológicos e descrever a percepção do enfermeiro acerca do processo de desospitalização em cuidados paliativos oncológicos.

\section{METODOLOGIA}

Trata-se de uma pesquisa descritiva e exploratória, com abordagem qualitativa, realizada em um Hospital Federal do Rio de Janeiro, nos setores de Internação Clínica, Cirúrgica e Emergência. A escolha por esta unidade hospitalar se deu em virtude do percentual de atendimentos oncológicos, uma vez que se trata de uma UNACOM (Unidade de Assistência de Alta Complexidade em Oncologia) e o alto percentual de pacientes em cuidados paliativos, dispondo ainda de um serviço de desospitalização estruturado e atuante na unidade.

Os participantes deste estudo foram 09 enfermeiros componentes da equipe multiprofissional, sendo utilizada como estratégia para a coleta de dados a realização de entrevistas com 14 perguntas guiadas por um roteiro semiestruturado, coletadas de modo individualizado, com duração média de trinta minutos, gravadas em aplicativo de celular e transcritas posteriormente. Os enfermeiros participantes eram os profissionais atuantes em cada plantão das unidades estudadas, considerando a sua

maior proximidade com a prática diária, sendo este um dos critérios de inclusão.

A população

deste estudo foi

constituída por

09 enfermeiros

atuantes nas diversas

áreas hospitalares

elencadas para o

estudo, com tempo

médio de Graduação

de 11 anos e 7

meses e atuação no

hospital de 04 anos

e 7 meses. Destes

profissionais, todos

apresentavam Curso

de Especialização

em áreas diversas,

porém nenhum com

Especialização em

Cuidados Paliativos

ou Oncologia.
O período de coleta de dados foi de novembro a dezembro do ano de 2018, após convite formal dos pesquisadores, aceite do profissional e assinatura de Termo de Compromisso Livre e Esclarecido (TCLE), obedecendo à Resolução n. ${ }^{\circ}$ 466, de 12 de dezembro de 2012, sendo este estudo aprovado pelo Comitê de Ética em Pesquisa (CEP) do Hospital Federal Cardoso Fontes sob parecer n ${ }^{\circ}$ 3.106.018.

Os critérios de inclusão utilizados foram: ser profissional enfermeiro atuante no plantão nos setores estudados, atuação profissional de no mínimo três meses em um dos serviços selecionadas para o estudo, não ter tirado licença nos últimos 30 (trinta) dias. Como critérios de exclusão foram utilizados: a negativa de participação na pesquisa; profissionais de saúde com afastamento por férias, licenças, ou atuação em cargos de chefia e rotina; lotação funcional fora dos setores propostos.

A análise de dados seguiu as três fases apresentadas para a análise de conteúdo, sendo estas ${ }^{(9)}: 1$. Leitura flutuante como o primeiro contato com o material; 2. Leitura aprofundada para exploração do material; 3. Tratamento dos resultados por meio das interpretações e inferência dos autores, confrontando o material empírico e a literatura produzida sobre o tema.

\section{RESULTADOS E DISCUSSÃO}

A população deste estudo foi constituída por 09 enfermeiros atuantes nas diversas áreas hospitalares elencadas para o estudo, com tempo médio de Graduação de 11 anos e 7 meses e atuação no hospital de 04 anos e 7 meses. Destes profissionais, todos apresentavam Curso de Especialização em áreas diversas, porém nenhum com Especialização em Cuidados Paliativos ou Oncologia.

A visão do enfermeiro acerca dos cuidados paliativos e desospitalização

Foi constatado através dos dados coletados que os enfermeiros do hospital estudado demonstravam uma visão acerca dos cuidados paliativos baseada unicamente no processo de finitude, correlacionando a terminologia "paliação" ao processo de morte, 
não identificando possibilidade de cuidados que possam proporcionar melhoria da qualidade de vida, atividade até os últimos momentos de vida, redução da dor, dentre outros pontos apontados pela literatura.

Ainda, foi possível identificar a frase "não há nada o que fazer", o que pode caracterizar a sensação de impotência do profissional, frente às patologias em que não se tenha a possibilidade de cura, esquecendo, por vezes, a essência do cuidar em enfermagem pautada no humano em todos os momentos do ciclo da vida.

Ao serem indagados acerca do processo de desospitalização e cuidados paliativos os enfermeiros se expressaram da seguinte forma:

"Os cuidados paliativos são aqueles para os doentes mais próximos a morte" (Profissional 3).

"É como se ele estivesse, de certa forma, esperando o tempo para falecer" (Profissional 7).

"Cuidados paliativos na minha concepção é conforto e fim de vida" (Profissional 1).

"Desospitalização em cuidados paliativos é a alta hospitalar para morrer em casa" (Profissional 2).

Os relatos demonstraram a dificuldade da equipe em distinguir cuidados paliativos e cuidados de fim de vida, o que pode estar diretamente atrelado à formação e pouca discussão acerca da temática nos espaços de assistência intra e extra-hospitalares.

Ademais, foi possível vislumbrarmos a preocupação da equipe acerca da manutenção do conforto e qualidade de vida das pessoas com câncer em cuidados paliativos, mesmo diante da confusão entre finitude e paliação, percebe-se a preocupação com a pessoa e seus familiares nas falas dos profissionais.

"São pacientes que, de certa forma, já não tem mais perspectiva de melhora do quadro clínico dele e também não se tem muita coisa para fazer [...] é como se ele estivesse, de certa forma, esperando o tempo para morrer" (Profissional 5).

"CP são os cuidados que na verdade o paciente já não tem mais um prognóstico para uma cura, um tratamento, que a gente está somente dando um conforto [...]. O nosso foco principal é o conforto para o paciente e a família também até $o$ momento do óbito" (Profissional 6).

"CP é um conjunto de ações que ajudam o paciente a ter uma morte mais digna, já que não vão conseguir fazer nenhuma medida terapêutica efetiva para tratar a doença, a gente tenta proporcionar o maior conforto possivel para essa passagem dele, de modo tranquilo, sem dor, sem nada" (Profissional 28).

Ainda, alguns profissionais sequer conseguiam conceituar cuidados paliativos, o que pode demonstrar falta de aproximação teórica com a temática ou mesmo ausência de formação, mesmo que básica, com relação ao assunto, mesmo efetuando o seu cotidiano prático em uma Unidade de Alta Complexidade em Oncologia, com alta demanda de pessoas em CP.

Com relação ao processo de desospitalização, observou-se que este era entendido como um mecanismo de alta hospitalar ou mesmo dispositivo para redução de tempo de permanência na unidade sem ser apontado os benefícios para o doente e família em CP.

"Desospitalização é dar alta do hospital, não é?!" (Profissional 7).

"É esse negócio para o paciente não ficar muito tempo internado, aí eles dão logo alta quando não tem mais jeito" (Profissional 20).

Neste contexto, observou-se ainda o desconhecimento por parte da equipe não médica acerca dos mecanismos e dispositivos da desospitalização existentes na pró- pria unidade de trabalho, o que por vezes pode ser um indicativo de não garantia de autonomia e escolha da pessoa acerca do local de cuidado, bem como do comprometimento do processo que depende do envolvimento de toda equipe.

"Desospitalização não sei te responder direito, porque isso é mais dos médicos" (Profissional 21).

"Desospitalização é o SADES, sempre envio pacientes para lá quando preciso dar alta e vejo a necessidade de continuidade de cuidados em domicílio" (Profissional 24).

\section{Possibilidades e limites da atuação do enfermeiro no processo de desos- pitalização da pessoa com câncer em cuidados paliativos}

Frente aos dados coletados, foi possível perceber potencialidades apresentadas pela equipe multiprofissional, bem como as limitações ao desempenhar as suas atividades no cenário estudado, sobretudo, no tocante ao processo de desospitalização em cuidados paliativos.

"Todos os dias atendemos pessoas em cuidados paliativos e que não querem permanecer no hospital" (Profissional 15).

"A postura profissional e abordagem frente aos CP: verdades e limitaçôes" (Profissional 18).

"Limitações e possibilidades em CP e desospitalização" (Profissional 8).

A percepção da vontade da pessoa com câncer em cuidados paliativos acerca do seu desejo de ir para o domicílio foi relatada pela equipe multiprofissional, em especial pela equipe de enfermagem que lida diariamente e diretamente com os cuidados e família durante as 24 horas do funcionamento hospitalar.

\footnotetext{
"Vejo pessoas dizendo que preferiam estar em casa e que sentem falta da
} 
sua família, da sua cama, das suas coisas, difícil" (Profissional 14).

Mesmo frente às falas, a dicotomia ente a solicitação do doente e a percepção da equipe acerca da autonomia e da possibilidade de implementação do processo de desospitalização ainda são limitados e precisam ser aprimorados na unidade.

Apesar da fragilidade de implementação de processos mais formais e efetivos de desospitalização e cuidados paliativos que parece ser um desafio para os profissionais, estes ainda reconhecem a existência de pessoas em paliação no seu atendimento diário, sendo necessária a humanização do cuidado prestado pela equipe, estando mais evidente na fala dos profissionais não médicos como podemos observar nos depoimentos abaixo.

"Sempre me apresentando como profissional e sempre falando diretamente a verdade" (Profissional 14).

"Conversar, procurar saber como é que está acontecendo naquele dia, é só o que podemos fazer pois eu acho que o nosso trabalho é muito mais com a familia" (Profissional 8).

Apesar de observarmos a necessidade de comunicação no processo de desospitalização em cuidados paliativos pelos profissionais não médicos da equipe multiprofissional, torna-se evidente a preocupação médica com a medicalização com vistas ao controle sintomático, o que por vezes pode retardar a implementação da desospitalização pelo paradigma do cuidado hospitalocêntrico.

"Quando se trata de um paciente em cuidados paliativos, visando o paciente e familiares, tento amenizar o máximo o que eu puder seja com relação à dor, sintomas, ou complicações que possam aparecer e quando necessário encaminho ao SADES" (Profissional 22).

Percebe-se na fala dos participantes o conhecimento acerca do setor de desos- pitalização existente no hospital, porém a compreensão acerca do termo "desospitalizar" ainda não parece esclarecida, estando este, por vezes, apenas vinculado ao encaminhamento de um setor para outro.

Ainda é possível observar que diante da não compreensão efetiva do termo "desospitalização", este pode ser encarado apenas como alta e confundido com modalidades terapêuticas que não se adequam ao caso, sobretudo, nos cuidados paliativos oncológicos.

"Desospitalização é quando o paciente começa a ser desmamado de algumas drogas que podem ser utilizadas, vamos dizer, somente $I V$. Eles vão começar a introduzir V.O e vão começar a trabalhar tanto no psicológico do paciente, quanto o da família ao fato dele poder voltar pra casa[...]" (Profissional 7).

Ademais, ter a clara compreensão do momento de início dos cuidados paliativos mesmo frente à dificuldade de definição de termos foi possível de ser observada, como é possível visualizarmos nas falas.

"Deve ser iniciada no momento que o paciente entra em cuidados paliativos e que tenha realmente condições de ir pro lar [...]. Eu acho que é perfeito" (Profissional 2).

"Eu entendo que essa desospitalização é necessária e possivel o mais rápido possivel, principalmente, para o paciente quando ele pode ter a oportunidade de estar perto dos seus familiares. Porque também tem essa equipe, que aqui no caso é o SADES que faz o serviço e que vai no domicílio" (Profissional 5).

"Deve ser iniciado tão logo que a pessoa perceba que o cuidado hospitalar não está mais trazendo benefício" (Profissional 22).

Neste contexto e mesmo frente à confusão de termos no que se refere a cuidados paliativos e cuidados de fim de vida, bem como desospitalização e alta hospitalar, os profissionais participantes deste estudo apontam benefícios do processo de desospitalização em cuidados paliativos no que se refere à participação da família, articulação e maior aproximação família e equipe, comunicação eficaz, treinamento de cuidador e maior qualidade de vida para pessoa com câncer e seus familiares.

Após a transcrição, leitura e análise das entrevistas com os profissionais participantes deste estudo, foi possível perceber o olhar desses profissionais sobre cuidados paliativos e o processo de desospitalização.

Percebe-se que mesmo as áreas em que essas pessoas atuam em diferentes cenários, o reconhecimento da existência de um crescente número de pessoas em paliação, sobretudo oncológicos, o que corrobora com os apontamentos das literaturas nacional e mundial ${ }^{(10-12)}$.

Mesmo frente a esta crescente, é possível perceber a precariedade no tocante à capacitação profissional, que reflete-se através da dificuldade na definição dos termos "Cuidados Paliativos" e "Desospitalização". Assim, acredita-se que os dados apontados pela literatura ${ }^{(12)}$ que enfocam a necessidade de capacitação dos profissionais de saúde acerca desta temática podem ser confirmados através das entrevistas realizadas.

No que tange à compreensão da desospitalização como um processo, ainda traz consigo entraves que dificultam esta implementação, seja por desconhecimento da equipe multiprofissional, seja por fragilidade no desenvolvimento do processo.

Nessa perspectiva, pode-se pressupor que a diversidade e complexidade de situações envolvidas no atendimento da pessoa com câncer em cuidados paliativos e sua família, por parte da equipe multiprofissional, geram desafios para a formulação e implementação de estratégias e ações facilitadoras ao processo de transição da unidade familiar do ambiente hospitalar para o ambiente domiciliar.

Portanto, pensar em desospitalização, nesse contexto, remete à ideia de que introduzir mudanças para a solução de problemas organizacionais, que requer pensar em soluções coletivamente, considerando que a 
possibilidade de inovações e mudança de paradigmas se constituem num processo sistêmico na medida em que envolve crenças, hábitos e interesses de indivíduos e grupos ${ }^{(13)}$.

No que concerne as inquietações da equipe, percebe-se que o cotidiano de trabalho é permeado pelos desafios de lidar com elementos da dimensão subjetiva, em que as ações de cuidado são marcadas por mobilizações mútuas de angústia, sofrimento e incertezas vivenciadas diante de sintomas resultantes do agravamento do câncer e da proximidade da morte.

O consenso é de que o modo de organização da atenção adotado na instituição do estudo está configurado a partir de uma lógica que, muitas vezes, desfavorece as inter-relações entre os profissionais da equipe da desospitalização com os profissionais dos outros setores, o que aponta, dentre as fragilidades, a comunicação para otimização deste processo.

$\mathrm{O}$ entendimento de que essas inter-relações compõem uma micropolítica, possibilita compreender as demandas dessa "realidade organizacional", ou seja, o conjunto de relações estabelecidas pelos vários atores organizacionais, formando uma rede complexa, móvel e mutante. Um campo marcado por disputas, acordos e composições, coalizões e afetos que permeiam o cuidado ${ }^{(14)}$.

Possivelmente, o olhar atento para fatores que estão relacionados tanto para a internalidade quanto para a externalidade dessa micropolítica, seja o aspecto a ser revisitado na organização do processo de trabalho para possibilitar as inovações e possíveis capacitações para adequações e apropriação de conceitos.

$\mathrm{Na}$ América Latina, as diretrizes para cuidados paliativos são orientadas pela Organização Pan-Americana de Saúde (OPAS) desde 2010, contudo, uma análi$\mathrm{se}^{(15)}$ acerca do desenvolvimento da desospitalização para os cuidados paliativos no Brasil, Argentina e Uruguai revelou que, tais diretrizes ainda se apresentam de modo incipiente, sendo mais desenvolvida como atendimento domiciliar no sistema privado de saúde e pouco estimulada como transição, sobretudo, no setor público de saúde,

\section{Possivelmente,}

o olhar atento

para fatores que

estão relacionados

tanto para a

internalidade

quanto para a

externalidade dessa

micropolítica,

seja o aspecto

a ser revisitado

na organização

do processo de

trabalho para

possibilitar

as inovaçôes

e possíveis

capacitaçôes para

adequações e

apropriação de

conceitos. o que vai de encontro com os depoimentos dos participantes deste estudo.

Assim, apesar do investimento nas políticas públicas visando atender às diretrizes internacionais para a assistência das pessoas em cuidados paliativos oncológicos, o modelo de desospitalização que vem sendo utilizado em nosso país ainda apresenta fragilidades, no que tange às diretrizes específicas para nortear essa prática, bem como a definição de termos e a capacitação profissional para a compreensão desta prática como processo iniciado ainda no ambiente hospitalar e com a participação de profissionais de saúde de diferentes setores envolvidos no atendimento da pessoa e família.

Deste modo, acredita-se que a recente publicação da Resolução n.o 41, de 31 de outubro de 2018, que trata das diretrizes para organização dos cuidados paliativos, à luz dos cuidados continuados integrados nos diferentes pontos da rede de atenção à saúde, possa colaborar para ampliar discussões que favoreçam a reestruturação dos processos de trabalho nas diferentes instituições que integram a rede de atenção à saúde.

Desta forma e visando atender os elementos apontados pela equipe multiprofissional no que tange à comunicação e benefícios para a família e pessoa em cuidados paliativos oncológicos com a implementação do processo de desospitalização, penso que este esteja para além da simples retirada da pessoa do hospital, e sim como um ato de continuidade das ações iniciadas no ambiente intra-hospitalar, bem como a manutenção da articulação em rede, de modo integrado e humanizado ao implementar ações pactuadas com a unidade familiar.

\section{CONCLUSÃO}

Ao se iniciar a pesquisa, percebeu-se que os profissionais apresentavam dificuldades de compreensão, acerca dos termos "cuidados paliativos" e "desospitalização", sendo estes comumente confundidos com conceito de "finitude da pessoa com câncer" e "alta hospitalar".

Este trabalho levou à percepção da existência de uma lacuna teórica ou acadêmica que os profissionais entrevistados apresen- 
tam, mesmo diante do atendimento diário de um alto índice de pacientes em cuidados paliativos oncológicos. Acredita-se que a prática cotidiana do cuidar e a crescente demanda de pessoas com câncer em cuidados paliativos torna cada vez mais necessária a formulação de estratégias para proporcionar embasamento teórico e capacitação da equipe multiprofissional, colaborando com a construção de práticas mais qualificadas embasadas em princípios científicos e com clareza de definições conceituais.

Ainda se evidenciou que, mesmo frente ao atendimento frequente de pessoas com câncer em cuidados paliativos na unidade, falta a identificação quanto aos serviços que a própria instituição oferece, o que pode dificultar a autonomia e escolha destas pessoas quanto ao local de cuidado e a participa- ção da família de modo ativo na condução dos processos de desospitalização.

Tal fato engloba uma necessidade de otimização dos processos de trabalho pautados na comunicação e humanização do cuidado, fornecendo um atendimento integrado e articulado com vistas ao atendimento das necessidades da população atendida.

Ademais, o estudo evidencia a necessidade de ampliação de estudos acerca da temática abordada visando contribuir com a atualização e capacitação profissional, bem como com a melhoria da qualidade da assistência e favorecer com o aprimoramento das diretrizes nacionais, melhorando as políticas intra e extra-hospitalares de atenção à pessoa em $\mathrm{CP}$ com possibilidade de ser desospitalizada.

Acredita-se também que as limitações deste estudo estejam presente, pois englo- bam os profissionais de apenas uma instituição, o que demonstra uma única realidade, podendo não ser a mesma em se tratando de outras unidades de saúde, mas que possibilita uma reflexão acerca da temática, fomentando a construção de novos estudos e favorecendo a ampliação de discussões em outras instituições do setor saúde.

Conclui-se que, apesar das ampliações acerca das discussões que permeiam a temática dos cuidados paliativos, em virtude da crescente de demanda de pessoas apresentando tais condições, sobretudo os oncológicos, compreender o enfoque da equipe sobre o tema nos possibilita refletir a prática sobre a sua própria prática, possibilitando a construção de estratégias e inovações que favoreçam a melhoria da qualidade assistencial. -

\section{REFERÊNCIAS}

1. Ministério da Saúde, Secretaria de Atenção à Saúde, Departamento de Atenção Básica (BR). Cadernos de Atenção Domiciliar [Internet]. Brasília (DF): Ministério da Saúde, 2012 [acesso em 27 mai 2019]. Disponivel em: http://saude.gov.br/editora.

2. Silvia KI, et al. Trajetória Política de Atenção Domiciliar em Minas Gerais. REME [Internet]. 2019 [acesso em 01 dez 2019]; 23. DOl: http://www.dx.doi.org/10.5935/14152762.20190002.

3. Olario OS, Moreira MC, Moreira IB, Martins JCA, Souza AT. Desospitalização em cuidados paliativos: Perfil dos usuários de uma unidade no Rio de Janeiro/ Brasil. Cogitare Enferm [Internet]. 2018 [acesso em 01 dez 2019]; 23(2):e53787. DOI: http://dx.doi.org/10.5380/ce.v23i2.53787.

4. Cordeiro FR, Kruse MHL. É possível morrer no domicílio? Análise dos cenários brasileiros e francês. Texto e Contexto [Internet]. 2019 [acesso em $01 \mathrm{dez}$ 2019]; 28. Disponível em: http://www.scielo.br/scielo.php?script=sci_arttext\&pid=S0104-07072019000100311\&Ing=en\&nrm=iso\&tlng=pt.

5. World Health Organization. How many people at the end of life need palliative care worldwide? Global Atlas of Palliative Care at the end of life [Internet]. Londres: WPCA, 2014 [acesso em 15 abr 2019]. Disponivel em: http://www.who.int/cancer/ publications/palliative-care-atlas/en/.

6. Merhy EE, Feuerwerker LCM. Atenção domiciliar: medicalização e substitutividade. In: Seminário Nacional de Divulgação dos Resultados da Pesquisa: implantação de atenção domiciliar no âmbito do SUS - modelagem a partir das experiências correntes [Internet]. n. 1, 2008, Rio de Janeiro. Anais eletrônicos... Rio de Janeiro: Universidade Federal do Rio de Janeiro, 2008 [acesso em 30 mai 2019]. Disponivel em: http://www. medicina.ufrj.br/micropolitica/pesquisas/atencaodomiciliar/

\section{textos/ad-}

edicalizacao_e_substitutividade.pdf.

7. Conselho Federal de Enfermagem (BR). Resolução COFEN n. ${ }^{\circ}$ 0564/2017 Aprova o novo código de ética dos profissionais de enfermagem [Internet]. 2017 [acesso em 01 dez 2019]. Disponivel em: http://www.cofen.gov.br/resolucao-cofen-no5642017_59145.html.

8. Minayo MCS. Pesquisa social: teoria, método e criatividade. 21. Ed. Petrópolis: Vozes; 2010.109p.

9. Bardin L. Análise de conteúdo. Lisboa: Edições 70; 2011.

10. Ministério da Saúde, Instituto Nacional de Câncer (BR). Estimativa 2018: incidência de câncer no Brasil [Internet]. 2018 [acesso em 25 mar 2019]. Disponivel em: http://www.inca.gov. br/estimativa.

11. World Heaslth Organization. Global Atlas of Palliative Care at the End of Life. WHO, 2014.

12. Silva MM, Buscher A, Moreira MC, Durte SCM. Visitando hóspices na Alemanha e no Reino Unido na perspectiva dos cuidados paliativos. Esc Anna Nery Rev Enferm. 2015 abr./ jun.; 19(2):369-375.

13. Motta PR. Gestão Contemporânea: A Ciência e a Arte de ser dirigente. 15. ed. Rio de Janeiro: Record; 2004.

14. Cecílio LCO. Apontamentos teórico-conceituais sobre processos avaliativos considerando as múltiplas dimensões da gestão do cuidado em saúde. Interface: Comunic., Saúde, Educ. 2011; 15(37):588-589.

15. Simão VM, Mioto RCT. 0 cuidado paliativo e domiciliar em países da América Latina. Saúde Debate. 2016; 40(108):156169. 